\title{
Conservative Management of Calcaneal Fractures. A Retrospective Review of Treatment Outcome
}

\author{
HY Wong, MD, AS Vivek*, FRCS (Edin), BC Se To, FRCS (Edin) \\ Department of Orthopaedics and Traumatology, Hospital Penang, Penang, Malaysia \\ * Department of Orthopaedic, University Malaya Medical Centre, Kuala Lumpur, Malaysia
}

\begin{abstract}
Introduction: Treatment of calcaneal fracture is still controversial and indication for surgery is not well established. We are reporting the mid term outcome of calcanel fractures treated conservatively.
\end{abstract}

Material and Methods: Patients admitted with calcaneal fractures from 1st November 2002 till 31st December 2004 and were treated conservatively were included in this study. The fractures were grouped according to Essex-Lopresti classification and their outcomes were assessed with the Maryland foot score. We also looked at time to weight bearing and returning to occupational activity.

Results: Forty-four patients were included for evaluation. Patients with extraarticular calcaneal fractures had significantly higher rating scores compared to those with intraarticular fractures (98.2 and 88.8 respectively, with a $\mathrm{p}$ value $=0.0001)$. Generally, both group of patients had a good clinical outcome. 18 of the 44 patients (41\%) started partial weight bearing before or at 6 weeks and 31 patients (72\%) were able to start full weight bearing by 12 weeks. 31 patients $(72 \%)$ were back to work 12 weeks after the injury.

Conclusion: Conservative management for calcaneal fractures is an acceptable mode of treatment with satisfactory functional outcome.

Key Words:

Calcaneal fractures, Conservative.

\section{INTRODUCTION}

The calcaneus (os calcis) is the commonest tarsal bone to sustain fracture. It accounts for $2 \%$ of all fractures. Despite the advances in imaging and surgical techniques, management of this fracture is still a challenging task and the outcome of operative treatment remains uncertain ${ }^{1}$. The aim of this study is to evaluate the clinical outcome of both extraarticular and intraarticular calcaneal fractures that have been treated conservatively. We would like to look at the functional aspect of the treatment especially the duration required for them to bear weight and start walking.

\section{MATERIALS AND METHODS}

We retrospectively reviewed all patients admitted to our centre with calcaneal fractures who were treated conservatively from 1st November 2002 to 31 st December 2004. The cases were identified from the hospital admission record. Only patients with unilateral calcaneal fractures treated conservatively with a below knee plaster cast were included in this study. Cases of bilateral calcaneal fractures were excluded as it would be difficult to asses the scores for individual bone. The patients' medical folders and radiographs were traced from the records department and all patients were called back to our orthopaedic clinic for physical and functional evaluation.

The fractures were classified according to the Essex-Lopresti classification, which is based on plain radiographic findings at presentation ${ }^{2}$. We assessed the functional outcome with the Maryland foot score (Table I). In addition, we also looked at patient's employment, time required to start full weight bearing and duration of leave before returning to employment.

The statistical analysis was done with Stat view.

\section{RESULTS}

There were a total of 48 patients with calcaneal fractures treated during the study period. Four cases with bilateral calcaneal fractures were excluded. For the remaining 44 patients, there were $13(30 \%)$ extraarticular and $31(70 \%)$ intraarticular fractures. Mean age of the patients was 42 years old (ranged from 11 to 78 years). Most of them (61.4\%) were between 24 to 51 years old. There were 41 $(93.2 \%)$ males and $3(6.8 \%)$ females. Looking at the occupation, most $(65.9 \%)$ were blue collar workers. There were $3(6.8 \%)$ white collar workers and 12 patients $(27.3 \%)$ were unemployed. The mode of injury was fall in 25 cases (58.6\%) and motor vehicle accident in 19 cases $(43.2 \%)$. 
Table I: Maryland foot score

Pain
None: including sports
Slight: no change in ADLs
Mild: minimal change in ADLs
Moderate: significant decrease in ADLs
Marked: during minimal ADLs(e.g bathroom,simple house work
Stronger and frequent analgesics)
Disabled: unable to work or shop
Function
Gait: Distance walked
Unlimited
Slight limitation
Moderate (2-3 blocks)
Severe limitation (1 block)
Indoors only
Stablity
Normal
Weak feeling-no true giving way
Occasional giving way (1-2 months)
Frequent giving way
Orthotic device used
Support
None

Limp

None

Slight

Moderate

Severe

Unable to walk

Shoes

Any type

Minor concession

Flat, laced

With orthotics

Space shoes

Unable to wear shoes

Stairs

Normally

With banister

Any method

Unable

Terrain

No problem, any surface

Problems on stone, hills

Problems on flat surfaces

Cosmesis

Normal

Mild deformity

Moderate

Severe

Multiple deformities

Motion (ankle, subtalar, mid-foot, metatarsophalageal)

Normal

Slightly decreased

Markedly decreased

Ankylosed 


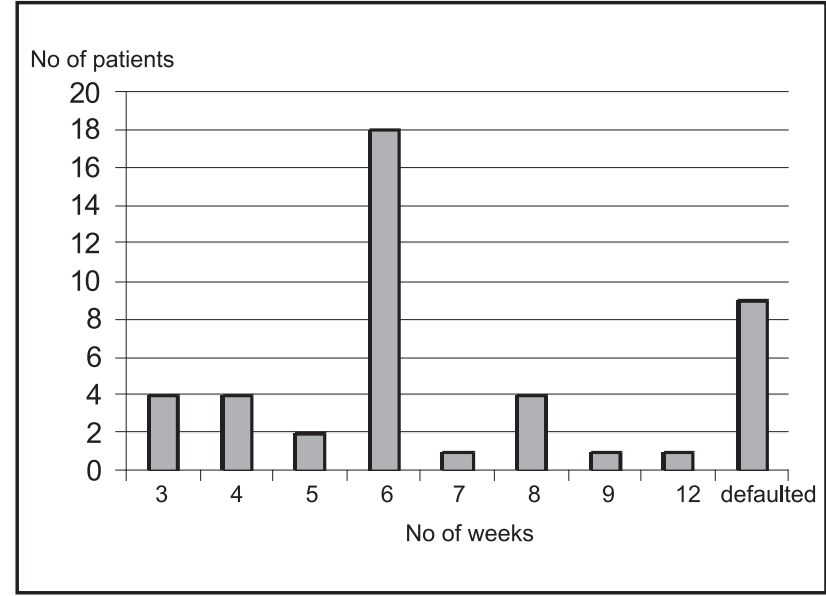

Fig. 1: Time duration when was POP removed.

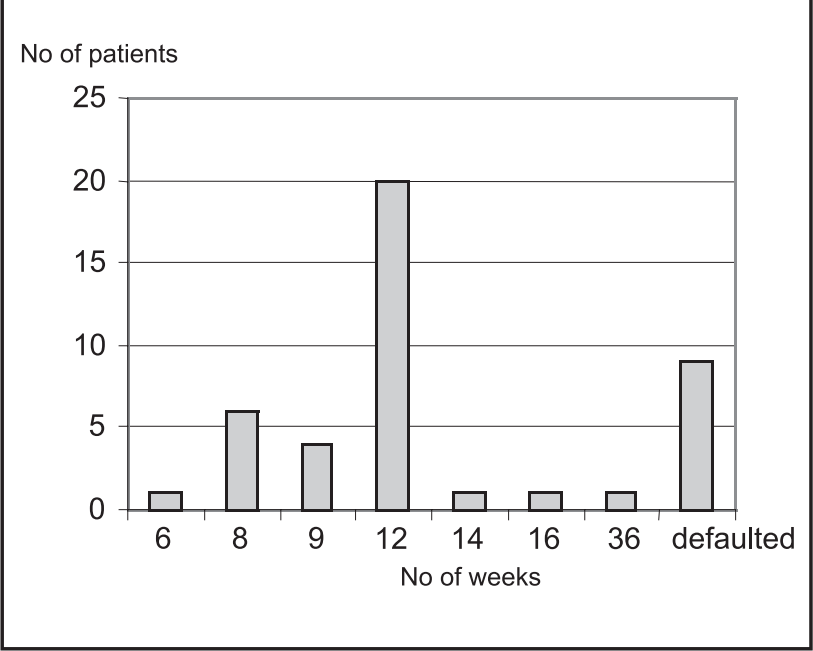

Fig. 3: Time duration for Return to Employment.

When we review the 6 week follow up records, 8 patients had defaulted. Out of the remaining 36 cases, 18 patients $(40.9 \%)$ had removed their cast by this period (Fig. 1). There were 10 patients who removed their cast earlier than 6 weeks against medical advice. By 12 weeks another patient had defaulted follow up. 31 patients $(72 \%)$ were able to fully weight bear at this time (Fig. 2). 31 patients (72\%) returned to work (including light duty work) by 12 weeks after injury, when they were able to start full weight bearing painlessly. $14 \%$ of the patients returned to work as early as 8 weeks after the injury (Fig. 3).

Generally, both groups of patients had good clinical outcome with the extraarticular fracture group doing better than the intraarticular calcaneal fracture group. Patients with extraarticular calcaneal fractures had significantly higher mean Maryland foot score (98.2) compared to those with intraarticular calcaneal fractures (88.8).

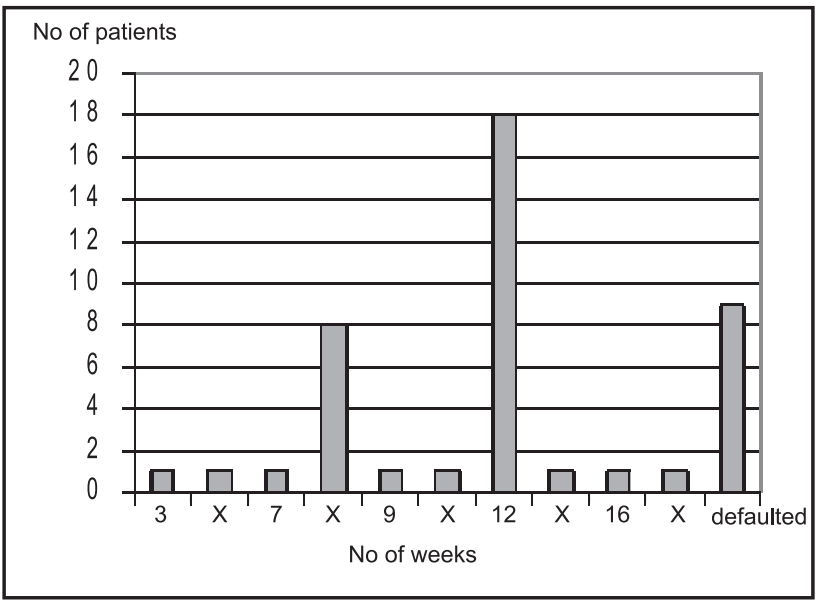

Fig. 2: Time duration to Full Weight Bearing.

\section{DISCUSSION}

The management of calcaneus fractures and their associated soft tissue injuries is a challenging task ${ }^{3}$. Being a cancellous bone, it may be deformed under compressive force during traumatic impact. Restoration of the anatomy is difficult as reduction and stabilization are technically demanding. Being a subcutaneous bone, most part of calcaneum is covered with a thin layer of skin and subcutaneous tissue. Injury to this area may be associated with damage to this soft tissue envelope and devascularization of the bone ${ }^{4}$. Surgical intervention could lead to wound breakdown and infection. The calcaneum forms 3 joints with the surrounding bones, namely the talo-calcaneal (talus and the calcaneum), calcaneal-cuboid (calcaneal and cuboid) and the talocalcaneal-navicular (talus, calcaneum and navicular) ${ }^{5}$. Most fractures involving the calcaneum will have a direct or indirect effect to these joints and their functions. Therefore, only an anatomical restoration of the fractured calcaneum to the preinjury state will avoid joint incongruity and complications such as secondary osteoarthritis. Considering the complexities of calcaneum and its associations, we believe that this is difficult to achieve with operative means.

In our centre, we tend to treat the calcaneal fractures nonoperatively with a below knee cast. We do not routinely request for Computer Tomography Scan ${ }^{6,7}$. For this study, we used the Essex-Lopresti classification ${ }^{2}$, which is based on the plain radiographs of the calcaneum. In developing countries with high percentage of its work force in the bluecollar category, many patients with calcaneal fracture will not be able to return to his or her occupation during the period of treatment. Therefore, it's an economic lost to the patient and their workplace. Our study shows that with conservative treatment, most of the patients were able to return to work at 12 weeks after the injury.

Both intraarticular and extraarticular calcaneal fractures treated conservatively can achieve a satisfactory functional result. Those with extraarticular fractures generally did 
better than those with intraarticular fractures. Richard et al ${ }^{8}$ reported that without stratification of the groups, the functional results after nonoperative care of displaced intraarticular calcaneal fractures were equivalent to those after operative care. However, after unmasking the data by removal of the patients who were receiving Workers' Compensation, the outcomes were significantly better in some groups of surgically treated patients.

\section{CONCLUSION}

In conclusion, we found that conservative management for both intra and extra articular calcaneal fractures remains an acceptable mode of treatment with a satisfactory functional outcome based on this mid term review. This is a cost effective option especially for hospitals with limited financial resources and operating facilities. 


\section{REFERENCES}

1. Rockwood and Green's Fractures In Adults 4th edition, Vol 2, Chap. 32 Fractures and Dislocations of the Foot, edited by James D. Heckman.

2. Essex-Lopresti P: The Mechanism, Reduction Technique, and Results in Fractures of the Os Calcis. Br. J. Surg 1952; 39: $395-419$.

3. Sanders R, Fortin P, DiPasquale T, Walling A: Operative Treatment in 120 Displaced Intraarticular Calcaneal Fractures. Clin. Orthop. 1993; 290: 87-95.

4. Thornes BS, Collin AL, Timlin M, Corrigan J. Outcome of calcaneal fractures treated operatively and non-operatively. The effect of litigation on outcomes. Ir J Med Sci. 2002; 171: 155-7.

5. Guyer BH, Levinsohn EM, Fredrickson BE, Bailey GL, Formikell M. Computed Tomography of Calcaneal Fractures: Anatomy, Pathology, Dosimetry, and Clinical Relevance. Am J Radiol 1985; 145: 911-9.

6. Gilmer PW, Herzenberg J, Frank JL, Silverman P, Martinez S, Goldner JL. Computerized Tomographic Analysis of Acute Calcaneal fractures. Foot Ankle, 1986; 6: 184-93.

7. Lowrie IG, Finlay DB, Brenkel IJ, Gregg PJ. Computerized Tomographic Assessment of the Subtalar Joint in Calcaneal Fractures. J Bone Joint Surg 1988; 70B: 247-50.

8. Richard B, Suzanne T, Robert M, Graham P. Operative Compared with Nonoperative Treatment of Displaced Intra-Articular Calcaeal Fractures. J Bone Joint Surg 2002; 84(A): 1733-44. 